\title{
Tumor-specific cytotoxic T lymphocyte activity determines colorectal cancer patient prognosis
}

\author{
Christoph Reissfelder, ${ }^{1,2}$ Slava Stamova, ${ }^{3}$ Christina Gossmann, ${ }^{3}$ Marion Braun, ${ }^{3}$ Andreas Bonertz, ${ }^{3}$ Ute Walliczek, ${ }^{1}$ Mario Grimm, ${ }^{1,3}$ \\ Nuh N. Rahbari, ${ }^{1}$ Moritz Koch, ${ }^{1,2}$ Maral Saadati, ${ }^{4}$ Axel Benner, ${ }^{4}$ Markus W. Büchler, ${ }^{1}$ Dirk Jäger, ${ }^{5}$ Niels Halama, ${ }^{5}$ \\ Khashayarsha Khazaie, ${ }^{6}$ Jürgen Weitz, ${ }^{1,2}$ and Philipp Beckhove ${ }^{3}$ \\ 'Department of Ceneral, Visceral and Transplantation Surgery, Ruprecht-Carls University, Heidelberg, Germany. ${ }^{2}$ Department for Visceral, Thoracic and Vascular Surgery, University Hospital Carl Gustav Carus, \\ Technische Universität Dresden, Dresden, Germany. ${ }^{3}$ Translational Immunology Department and ${ }^{4}$ Department of Biostatistics, German Cancer Research Center, Heidelberg, Germany. ${ }^{5}$ Department of Medical \\ Oncology, National Center of Tumor Diseases and University Medical Center Heidelberg, Heidelberg, Germany. ${ }^{6}$ Robert H. Lurie Comprehensive Cancer Center, Northwestern University, Feinberg School of \\ Medicine, Chicago, Illinois, USA.
}

\begin{abstract}
The composition of tumor-targeted T cell infiltrates is a major prognostic factor in colorectal cancer (CRC) outcome; however, the functional role of these populations in prolonging patient survival remains unclear. Here, we evaluated 190 patients with CRC for the presence of functionally active tumor-infiltrating lymphocytes (TILs), the tumor specificity of these TILs, and the correlation between patient TILs and long-term survival. Using intracytoplasmic cytokine staining in conjunction with HLA multimers loaded with tumor peptide and antigen-specific cytokine secretion assays, we determined that TNF- $\alpha$ expression delineates a population of tumor antigen-specific (TA-specific) cytotoxic T lymphocytes (CTLs) present within tumors from patients with CRC. Upregulation of TNF- $\alpha$ expression in TILs strongly correlated with an increase in the total amount of intratumoral TNF- $\alpha$, which is indicative of tumor-specific CTL activity. Moreover, a retrospective multivariate analysis of 102 patients with CRC, which had multiple immune parameters evaluated, revealed that increased TNF- $\alpha$ concentration was an independent prognostic factor. Together, these results indicate that the prognostic impact of $\mathrm{T}$ cell infiltrates for CRC maybe largely based on subpopulations of active TA-specific T cells within the tumor, suggesting causal implication for these cells in patient survival. Additionally, these results support the use of intratumoral TNF- $\alpha$, which is indicative of T cell function, as a prognostic parameter for CRC.
\end{abstract}

\section{Introduction}

Colorectal cancer (CRC) is the third most frequent malignancy worldwide. Each year there are approximately 1 million new cases of CRC and half a million deaths attributable to this disease (1). The treatment of choice remains surgical resection, which provides cure for a considerable number of patients (2). Nevertheless, the management of patients with CRC has improved substantially within the past 2 decades, owing to more effective chemotherapeutics as well as improved operative technique and perioperative care. The immune system came recently into focus as a new therapeutic option.

In 2006, Galon et al. demonstrated that high infiltrates with effector and memory T cells (TCs) correlate with improved relapse-free and overall survival in patients with CRC (3). Pages et al. confirmed that high numbers of tumor-infiltrating memory TCs in CRC are associated with the absence of pathologic evidence of early metastatic invasion and improved survival (4). Based on these pioneering studies and numerous subsequent investigations, TC infiltration has been meanwhile established as

Authorship note: Christoph Reissfelder, Slava Stamova, and Christina Gossmann contributed equally to this work.

Conflict of interest: The authors have declared that no conflict of interest exists. Submitted: December 20, 2013; Accepted: November 13, 2014.

Reference information: J Clin Invest. 2015;125(2):739-751. doi:10.1172/JCI74894. an independent prognostic factor in CRC, having an even stronger prognostic significance than conventional TNM staging (5-9).

Despite the undoubted relevance of TC infiltrates for CRC prognosis, it remains unclear whether TCs play a causal role in improved prognosis or merely characterize tumors with beneficial tumor cell biology. Clarification of the causal affect of spontaneous TC infiltrates on patient prognosis would not only enable the development of function-related immunologic biomarkers of higher prognostic and predictive accuracy but also strongly affect the perception and further development of TC-based cancer immunotherapy for CRC.

Tumor antigen-specific (TA-specific) TCs, including functionally potent tumor-reactive memory TCs that are capable of secreting effector cytokines upon TA encounter, are spontaneously generated in many patients with cancer, including those with CRC (10-13). TA-specific TCs can be detected in the blood and bone marrow of $30 \%$ to $40 \%$ of patients with CRC $(14,15)$. Also, strong TC accumulations in colorectal carcinomas are associated with oligoclonal expansions of tumor-infiltrating TCs - pointing to a potential selective enrichment of tumor-specific TCs in situ (16). On the other hand, the mere presence of tumor-specific cytotoxic $\mathrm{T}$ lymphocytes (CTLs) in the blood, as determined by HLA tetramer analyses, was not correlated with improved clinical outcome (17).

Tumors regulate TC recruitment through the expression of TC trophic chemokines and through influencing the activity of 
the respective tumor vasculature $(18,19)$. In addition, they regulate activation, maintenance, and activity of antigen-specific TCs through a plethora of immune-modulatory mechanisms, involving recruitment of immune-suppressive cell populations, particularly Tregs, MDSCs, or mast cells, among others; secretion of immune-suppressive cytokines, such as TGF- $\beta 1$; or expression of immune-modulatory ligands, such as PDL-1 (19-25). Regulation of TC activity in situ occurs at interindividually varying levels, and indeed, Koch et al. were able to demonstrate the tumor-selective activation and cytotoxic activity in situ of small, individually varying subpopulations of CD8 ${ }^{+}$TCs in CRC (14). Therefore, prognostic relevance of tumor-infiltrating lymphocytes (TILs) in CRC may be restricted to the subpopulation of in situ active, TA-specific effector TCs, and, as a consequence, the functional activity of tumor-specific TCs in CRC tissue might be a better parameter of CRC prognosis than mere TC infiltration.

Functional activity of TCs is characterized by their release of effector cytokines. Observations from preclinical tumor models and from human vaccination studies show that polyfunctional type 1 TCs simultaneously expressing TNF- $\alpha$, IFN- $\gamma$, and IL- 2 particularly correlated with immune protection, while TCs expressing IFN- $\gamma$ alone may not correlate with improved clinical outcome (26, 27). Cytokine secretion is induced by antigen-specific stimulation and, in the case of TNF- $\alpha$, is mainly restricted to the first $2-4$ hours after activation (28). We therefore hypothesized that increased intratumoral concentration of TNF- $\alpha$ indicates the presence of in situ active tumor-specific type 1 TCs and predicts improved prognosis of patients with CRC better than TC infiltration alone.

To investigate this hypothesis, here we studied the presence, antigen specificity, and functional activity of systemic and intratumoral effector TCs in 88 patients with CRC altogether. We show that, in patients with CRC, TNF- $\alpha$ expression in TILs is restricted to the subpopulation of activated TCs, that intratumoral TNF- $\alpha$ expression is only increased in patients containing tumor-reactive TCs, and that in TILs TNF- $\alpha$ expression is restricted to the population of tumor-specific TCs. Furthermore, we demonstrate that the total content of TNF- $\alpha$ in CRC tissue strongly correlates with the numbers of infiltrating TNF- $\alpha$-expressing TCs. Thus, intratumoral TNF- $\alpha$ concentration is a direct indicator of the in situ activity of tumor-specific effector TCs in CRC.

On this basis, we studied the prognostic relevance of intratumoral TNF- $\alpha$ in comparison to other established or proposed factors of CRC prognosis in a second cohort of 102 patients with CRC retrospectively. Besides TNM staging, these studies involved the infiltration by $\mathrm{CD}^{+}$and $\mathrm{CD}^{+}$conventional TCs (Tconv), Tregs and their related immune-suppressive effector cytokine TGF- $\beta 1$, and mast cells. While a prognostic relevance has been well established before for $\mathrm{CD}^{+}$and $\mathrm{CD} 8^{+}$Tconv, conflicting data exist regarding a potential adverse or protective role of tumor-infiltrating Tregs, which has been largely assigned to their potential suppression of tumor-specific TC responses or of protumorigenic cancer inflammation, respectively $(29,30)$. In contrast, mast cells, which exert proinflammatory activity in CRC, have been lately controversially discussed as potential protumorigenic mediators of poor prognosis $(22,31-33)$. Based on 10-year follow-up data, we demonstrate that intratumoral TNF- $\alpha$ levels represent the best prognostic criteria for 10-year overall survival in patients with
CRC. Thus, our study provides evidence of a direct functional involvement of TA-specific TC activity in improved prognosis of patients with CRC.

\section{Results}

TNF- $\alpha$ expression is restricted to activated tumor-infiltrating TCs. In a first set of experiments, we investigated by flow cytometry whether TNF- $\alpha$ characterizes a subpopulation of activated TCs in CRC tissue. $\mathrm{CD}^{+}$and $\mathrm{CD} 8^{+}$TILs from CRCs that rested overnight without stimulation showed no expression of TNF- $\alpha$, while subsequent polyclonal activation with anti-CD3/anti-CD28 antibodies caused marked TNF- $\alpha$ expression in $\mathrm{CD}^{+}$and $\mathrm{CD} 4^{+}$TILs (Figure $1, \mathrm{~A}$ and $\mathrm{B}$ ) and also in freshly isolated blood-derived TCs from 5 patients with CRC (Figure 1C). Altogether, activation-induced TNF- $\alpha$ expression was lower in TILs than in peripheral bloodderived TCs (PBTCs) from the patients - most likely reflecting the exhausted state of intratumoral TCs (34). Taken together, TNF- $\alpha$ expression in TILs is restricted to a population of activated TCs.

We next quantified TNF- $\alpha^{+}$TCs in CRC tissue by ex vivo flow cytometry immediately after tumor resection. TNF- $\alpha^{+} \mathrm{CD}^{+}$and $\mathrm{CD} 4^{+} \mathrm{TCs}$ were detectable in 4 of $8 \mathrm{CRCs}$, with relative proportions of $0.2 \%$ to $1.9 \%$ for $\mathrm{CD} 8{ }^{+} \mathrm{TCs}$ and of $0.7 \%$ to $3.1 \%$ for $\mathrm{CD} 4^{+} \mathrm{TCs}$, and were significantly enriched in CRC when compared with the normal colonic mucosa of the same patients (Figure 1, D and E). Notably, the absolute concentration of TNF- $\alpha$ was also significantly increased in a cohort of 14 primary CRC tissues $(0.4 \mathrm{pg} / \mathrm{ml})$ compared with the respective corresponding normal colonic mucosa $(0.1 \mathrm{pg} / \mathrm{ml})$ (Figure 1F). These data demonstrate for a subgroup of patients with CRC a tumor-selective accumulation of in situ active type 1 effector TCs in CRC tissues.

TNF- $\alpha$ expression in CRC infiltrating lymphocytes is restricted to tumor-specific TCs. We wondered whether the observed increase of TNF- $\alpha^{+}$TCs and TNF- $\alpha$ protein in some CRCs was based on endogenous tumor-specific TC responses in these patients. We therefore assessed the presence of functionally potent, tumor-specific TCs (hereafter referred to as "tumor-reactive") in the blood or bone marrow (which represents a preferred homing site of TA-specific memory TCs) (34-36) from 26 patients with primary CRC by an ex vivo 40-hour short-term IFN- $\gamma$ ELISPOT assay (15, $36,37)$ and compared the data to the respective amount of TNF- $\alpha$ protein in corresponding tumor tissue. To determine the presence of tumor-reactive TCs, purified $\mathrm{CD}^{+}$TCs were stimulated for 40 hours with autologous DCs, which were loaded with $200 \mu \mathrm{g} / \mathrm{ml}$ autologous tumor cell lysate or the same amount of autologous peripheral blood mononuclear cell (PBMC) lysate and/or lysate of autologous normal colonic mucosa as negative control antigen. Tumor-specific TC reactivity was assumed in cases in which IFN- $\gamma$ spot numbers in triplicate test wells significantly exceeded those in triplicate negative control wells $(P<0.05)$. In this assay format, IFN- $\gamma$ secretion is restricted to endogenous antigen-experienced effector/memory TCs $(15,38)$. The result of a representative experiment demonstrating the presence of tumor-reactive TCs in the blood of a patient with CRC is shown in Figure 2A. Altogether, we detected tumor-reactive TCs in $44 \%$ of the patients (Figure 2B). The detection rate was not influenced by differences in IFN- $\gamma$ background activity in the control wells, as these did not differ between tumor-reactive and unresponsive test samples $(15,34)$. 
A

Rested TIL (CRC)

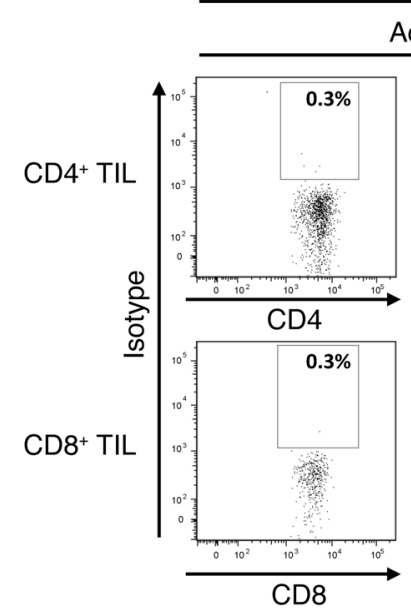

Activated

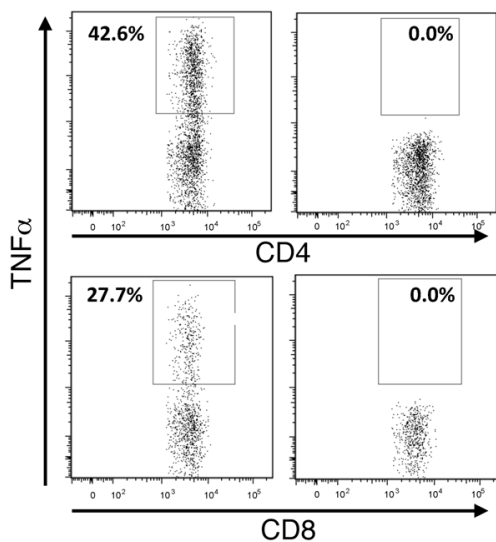

B Rested TIL (CRC)
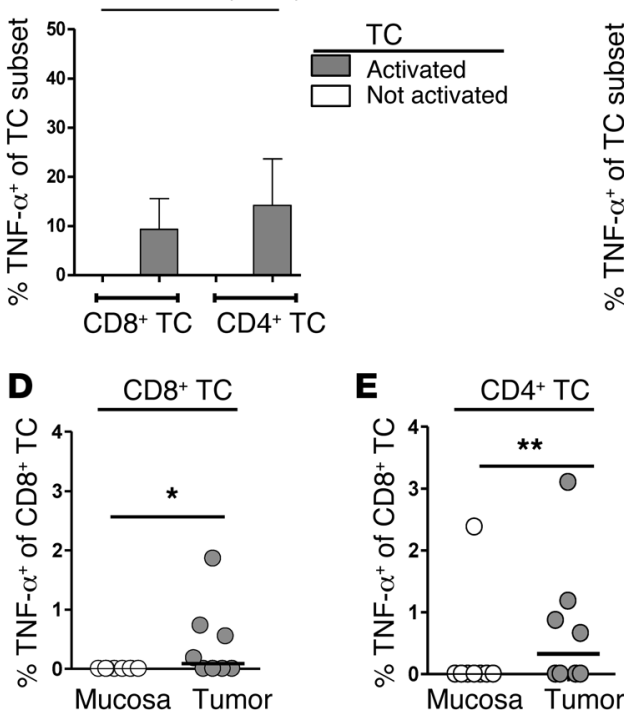
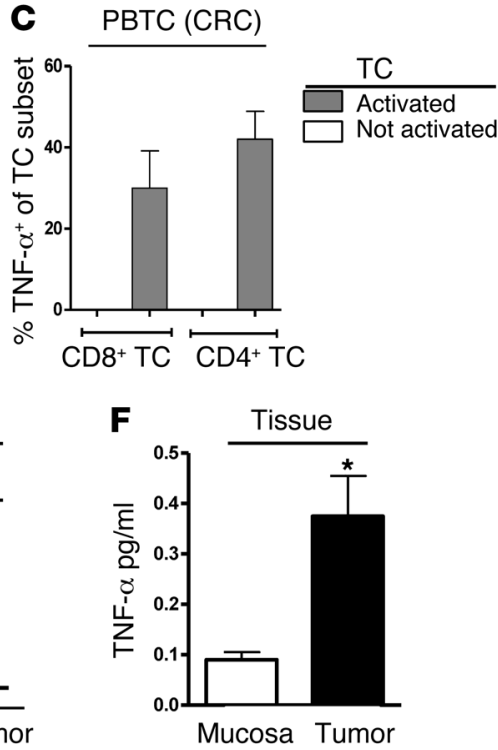

Figure 1. Increased TNF- $\alpha$ expression in activated TCs. (A) TNF- $\alpha$ expression in polyclonally activated (middle column) but not in unstimulated (right column) CD4+ and CD8 ${ }^{+}$TILs from one representative patient with CRC $(n=4)$ was detected by flow cytometry using TNF- $\alpha$-specific mAb or a respective isotype $m A b$ as negative control (left column). Numbers in dot plots indicate proportions (\%) of TNF- $\alpha$-positive cells among gated cells. (B and $\mathbf{C}$ ) Mean proportions of TNF- $\alpha$-expressing CD8 $8^{+}$or $C D 4^{+}$TCs in rested TILs from 4 patients with CRC (B) and in the blood of 5 patients with CRC (C), without (white bars) or after polyclonal stimulation (gray bars), are shown as the percentage of the respective TC subset. Before analysis, freshly isolated TILs were rested overnight without stimulation. Error bars show mean + SD. (D and E) Proportions of TNF- $\alpha$-positive $\mathrm{CD}^{+}$(D) or $\mathrm{CD} 4$ (E) TCs in freshly isolated TCs from CRC tissue (gray circles) or corresponding normal colonic mucosa (white circles) of 8 patients with CRC. Circles indicate values from individual samples. Mean values are indicated by black lines. (F) Concentrations of TNF- $\alpha$ protein in CRC tissue and corresponding normal colonic mucosa from 14 patients (mean \pm SEM). ${ }^{*} P<0.1,{ }^{* *} P<0.05$, as determined by 2 -tailed paired $t$ tests.

For an example of this, see data shown shown in Figure 2D, and cumulative data for PBTCs is shown in Figure 2E and that for paired peripheral blood and bone marrow samples is shown in Figure $2 \mathrm{~F}$. We found that, indeed, most patients contained both TA-reactive IFN- $\gamma$ - and TNF- $\alpha$-producing TCs. Interestingly, only small minorities of stimulated TCs secreted both cytokines at the same time, while the majority of TA-reactive TCs secreted either TNF- $\alpha$ or IFN- $\gamma$. In some patients, we found dominant TA-specific IFN- $\gamma$ secretion, while in others TNF- $\alpha$ secretion was dominant. Accordingly, intratumoral TNF- $\alpha$ and IFN- $\gamma$ lev-

TNF- $\alpha$ contents, as determined by ELISA, in CRC tissues from 15 patients lacking systemic tumor-reactive TCs were consistently very low and comparable to those of healthy mucosa $(0.2 \mathrm{pg} / \mathrm{ml}$ vs. $0.1 \mathrm{pg} / \mathrm{ml}, \mathrm{NS}$ ). We detected increased intratumoral TNF- $\alpha$ (reaching average levels of $2.5 \mathrm{pg} / \mathrm{ml}$ ) and significantly increased proportions of TNF- $\alpha$-expressing TILs (Supplemental Figure 1; supplemental material available online with this article; doi:10.1172/ JCI74894DS1) only in the group of patients with endogenous tumor-reactive TC responses, as inferred by IFN- $\gamma$ ELISPOT analysis (Figure 2C), and the frequencies of tumor-specific IFN- $\gamma$ secreting TCs significantly correlated with increased amounts of intratumoral TNF- $\alpha(P=0.02$, data not shown), suggesting that both TNF- $\alpha$ and IFN- $\gamma$-secreting TCs are simultaneously generated in patients with CRC. In order to assess, whether patients with tumor-specific IFN- $\gamma$ responses also exerted tumor-specific TNF- $\alpha$ responses, we analyzed the simultaneous secretion of both cytokines in antigen-stimulated TCs by flow cytometric cytokine capture assay using a combination of well-described long synthetic peptides derived from common CRC-associated antigens, mucin-1, Her-2/neu, carcinoembryonic antigen (CEA), p53, or heparanase (15) as test antigens, and human IgG as negative control antigens. els did not strongly correlate with each other (Supplemental Figure 2). Taken together, the observed tumor-selective increase of TNF- $\alpha$ in CRCs compared with normal colonic mucosa described in Figure $1, \mathrm{D}-\mathrm{F}$, can be assigned to the contribution of the subgroup of patients containing circulating tumor-reactive TCs in their blood.

Based on our observations that in TILs TNF- $\alpha$ expression was confined to early activated TCs and that increased amounts of intratumoral TNF- $\alpha$ were only found in patients harboring tumor-reactive TCs, we hypothesized that in CRCs TNF- $\alpha$ expression is restricted to the subpopulation of TA-specific TCs. We therefore stained $\mathrm{CD}^{+}$TCs from 15 patients with HLA-A2-positive primary and metastatic CRC with HLA-A2 pentamers loaded with respective epitopes of the CRC-associated antigens mucin-1, Her-2/neu, CEA, p53, or heparanase and analyzed their frequencies and TNF- $\alpha$ expression in the blood, bone marrow, and tumors by multicolor flow cytometry. To exclude false-positive results, we used HIVgag-loaded HLA-A2 pentamers as negative control and subtracted respective background values. A representative staining for tumor-infiltrating CEA-specific $\mathrm{CD}^{+}{ }^{+} \mathrm{TC}$ is shown in Figure 3A. Figure 3, B and C, and Supplemental Figure 3 show the frequencies of pentamer-binding $\mathrm{CD} 8^{+} \mathrm{TCs}$. Altogether, we 
A

$$
\begin{gathered}
\text { AFN- } \gamma \text { Elispot-Assay } \\
\text { (representative CRC patient) }
\end{gathered}
$$

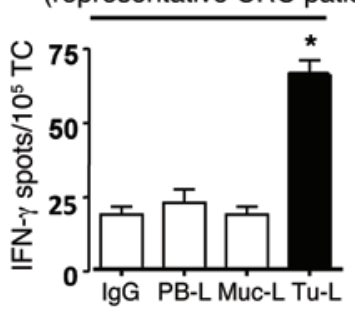

D

Cytokine capture-Assay (representative CRC patient)
B

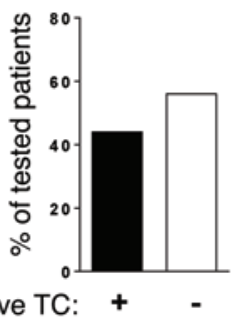

TA-reactive TC:
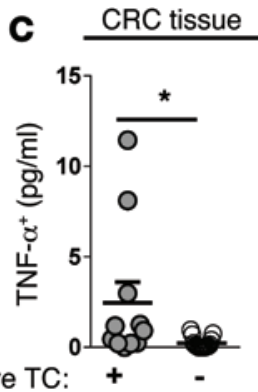

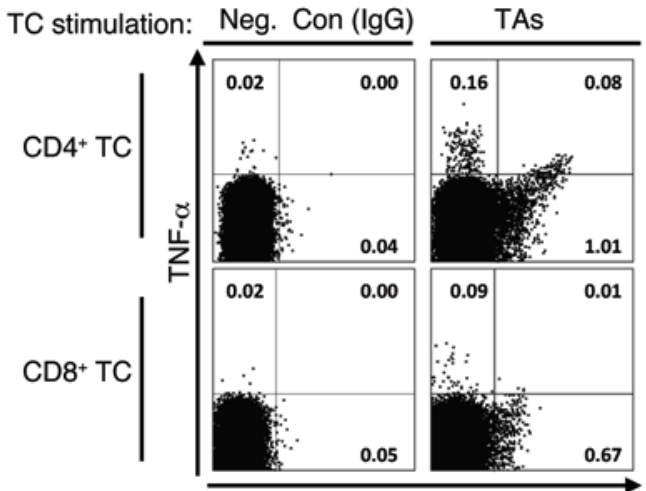

IFN- $\gamma$

\section{E Cytokine capture-Assay (cumulative data)}

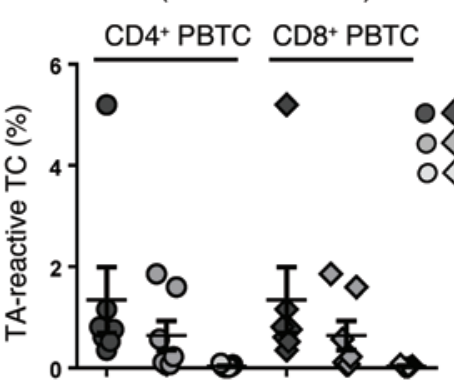
$\diamond$ IFN- $\gamma^{+}$TNF- $\alpha^{+}$

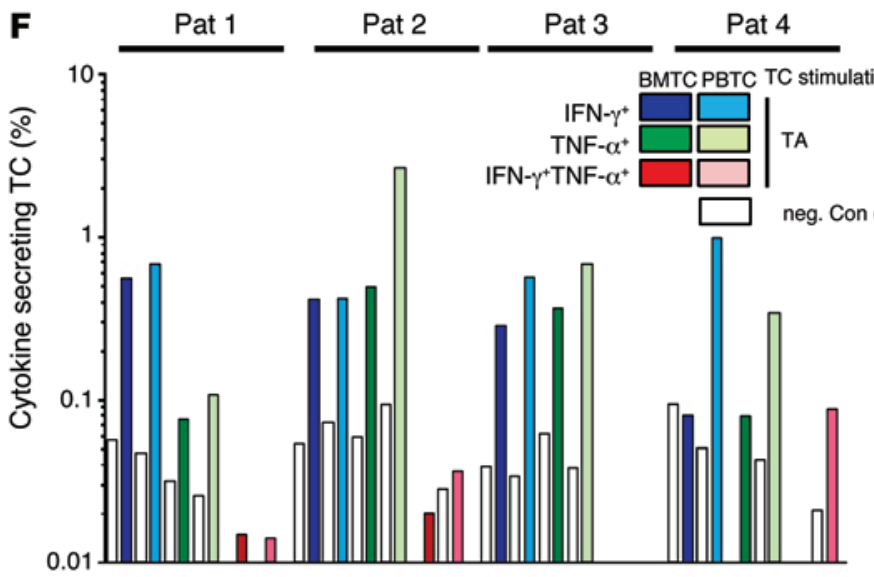

Figure 2. Increased TNF- $\alpha$ content in CRC tissue of patients with TA-reactive TC responses. (A) IFN- $\gamma$ ELISPOT from one representative patient with CRC $(n=3)$, showing IFN- $\gamma$ spot numbers (triplicates) from wells containing DCs pulsed with PBMC lysate (PB-L), normal colonic mucosa lysate (Muc- $L$ ), both autologous or human IgC (IgG) as controls (white bars), or with autologous tumor lysate (Tu-L, black bar). (B) Proportions of patients with CRC showing or lacking tumor-reactive (TA-reactive) TCs in peripheral blood or bone marrow $(n=26)$. (C) Absolute TNF- $\alpha$ protein concentration in CRC tumors from patients containing $(n=11)$ or lacking $(n=15)$ tumorreactive TCs in peripheral blood or bone marrow. Circles indicate values from individual patients. (D) Expression of IFN- $\gamma$ and TNF- $\alpha$ in gated $\mathrm{CD} 4^{+} \mathrm{T}$ and $\mathrm{CD} 8^{+} \mathrm{TC}$ in PBTCs stimulated with TA or with human IgG, as determined by cytokine capture assay. Data from one representative patient with CRC $(n=7)$ is shown. Numbers in dot plots indicate the proportion (\%) of cytokine-expressing cells among gated cells. (E) Proportion of cytokine-secreting TA-reactive $\mathrm{CD}^{+}$and CD8 ${ }^{+} \mathrm{TCs}$ in the blood of 7 patients with CRC. Each symbol represents data from an individual patient with the value of the background reactivity (against IgG) subtracted. (F) Proportion of cytokine-secreting TCs after stimulation with TA or IgG in the blood and bone marrow of 4 patients with CRC. ${ }^{*} P<0.05$, as determined by unpaired 2-tailed $t$ test; mean \pm SEM detected antigen-specific CD8 ${ }^{+}$TCs in 14 of 15 patients (ranging from $0.1 \%$ to $2.5 \%$ for individual antigens) and in 10 of $13 \mathrm{CRCs}$, without apparent differences between primary tumors and CRC metastases. Abundance and frequencies of specific TCs strongly varied among the individual patients. The most prominent TC target antigens were Her-2/neu and CEA (Figure 3C). Interestingly, the frequencies of pentamer-binding TCs were significantly increased in CRC tissue as compared with those in peripheral blood, suggesting that TC accumulation in CRCs involved antigen recognition (Figure 3B).

We next analyzed by intracytoplasmic cytokine staining the expression of TNF- $\alpha$ in pentamer binding and in unspecific CD $8^{+}$TCs. On average, TNF- $\alpha$ was expressed by $0.2 \%$ of antigen-unspecific (pentamer-negative) $\mathrm{CD}^{+}$TILs, while maximum proportions of TNF- $\alpha$-positive cells in this subgroup did not exceed $0.6 \%$ in single TIL cultures. In contrast, we detected a more than 20-fold increased frequency of TNF- $\alpha$-expressing cells in the subpopulation of TA-specific TILs, with an average frequency of $5.5 \%$ and maximum frequencies of up to $40 \%$ among $\mathrm{CD}^{+} \mathrm{CD}^{+}$pentamer ${ }^{+}$TILs (Figure 3D). Similarly, we also detected a significant increase of TNF- $\alpha$ expression in circulating and bone marrow-resident pentamer-binding $\mathrm{CD}^{+}$ TCs but not in antigen-unspecific TCs (Figure 3, E and F). Still, TNF- $\alpha$ was not expressed in pentamer-binding TCs from all patients but only in those from 9 of 14 patients and 4 of 10 CRCs. In these, TNF- $\alpha$ was expressed in $29 \%$ of antigen-specific TILs, $9 \%$ of antigen-specific PBTCs, and $16 \%$ of antigen-specific bone marrow TCs. In samples with high TNF- $\alpha$ expression of pentamer-binding TILs, we also predominantly detected high TNF- $\alpha$ expression in systemic TCs; however, in several cases with high peripheral TNF- $\alpha$ response, pentamer-binding TILs were TNF- $\alpha$ negative (Supplemental Figure 4). 


\section{Table 1. General patient characteristics}

\section{Patient characteristics} $N(\%)$

Gender

\begin{tabular}{|c|c|}
\hline Male & $62(61)$ \\
\hline Female & 40 (39) \\
\hline Age (yr) & $63(28-96)^{A}$ \\
\hline \multicolumn{2}{|l|}{ UICC stage } \\
\hline$\|$ & $43(42)$ \\
\hline III & $36(35)$ \\
\hline IV & $23(23)$ \\
\hline \multicolumn{2}{|l|}{$T$} \\
\hline 2 & $5(5)$ \\
\hline 3 & $80(78)$ \\
\hline 4 & $17(17)$ \\
\hline \multicolumn{2}{|l|}{ N } \\
\hline 0 & $49(48)$ \\
\hline 1 & $25(25)$ \\
\hline 2 & $28(27)$ \\
\hline \multicolumn{2}{|l|}{ Grading } \\
\hline I & $2(2)$ \\
\hline ॥ & $71(70)$ \\
\hline III & $29(28)$ \\
\hline \multicolumn{2}{|l|}{ Operative procedure } \\
\hline Right hemicolectomy & $22(21)$ \\
\hline Left hemicolectomy & $21(21)$ \\
\hline Sigmoid resection & $13(13)$ \\
\hline Rectum resection & $37(36)$ \\
\hline Rectum extirpation & $9(9)$ \\
\hline
\end{tabular}

Taken together, TNF- $\alpha$ expression was restricted to tumor-specific TCs, but pentamer-binding TILs expressed TNF- $\alpha$ only in a subgroup of $40 \%$ of patients. This is consistent with our observation that only a subgroup of approximately $46 \%$ of the patients developed systemic tumor-reactive type $1 \mathrm{TC}$ responses and that only this subgroup showed increased expression of TNF- $\alpha$ in CRCs.

Total TNF- $\alpha$ expression in CRC is determined by TNF- $\alpha$-expressing TILs. TNF- $\alpha$ is not exclusively expressed by activated TCs but can also be secreted by innate immune cells in the tumor microenvironment, such as activated macrophages, and by tumor cells (39). Nevertheless, TNF- $\alpha$ expression in innate immune cells can depend on the presence of activated TCs that trigger TNF- $\alpha$ production, e.g., in macrophages through CD40L/CD40 interactions. We studied the relation of TNF- $\alpha$-expressing TCs to TNF- $\alpha$ secretion by other cells by flow cytometry of freshly isolated tumor cell suspensions from 27 primary and 9 metastatic CRCs. TNF- $\alpha$ expression strongly correlated between $\mathrm{CD}^{+}$and $\mathrm{CD} 8^{+} \mathrm{TCs}$ of the same patients (Supplemental Figure 5), suggesting that tumor-specific CTL and type $1 \mathrm{CD}^{+} \mathrm{TC}$ responses mostly occur simultaneously. In primary tumors, the average proportions of TNF- $\alpha^{+}$TCs among $\mathrm{CD} 4^{+}$and $\mathrm{CD}^{+}$TCs were $0.23 \%$ and $0.27 \%$, respectively (Figure $4 \mathrm{~A}$ ), while TNF- $\alpha$ expression in TILs derived from CRC metastases was markedly reduced (0.07\% and $0.08 \%$, respectively) (Figure $4 \mathrm{~A})$.
Altogether, TCs constituted $17 \%$ and $6 \%$ of the entire TNF- $\alpha-$ expressing cell population in primary and metastatic tumors, respectively (Figure 4, B and C). In order to verify that TNF- $\alpha$ is spontaneously produced by tumor-infiltrating TCs in situ and to eliminate the possibility of differential cell activation or elimination during tissue dissociation, we also analyzed TNF- $\alpha$ expression in CRC tissue in situ by immunofluorescence microscopy. As shown in Figure 4D, we could confirm the presence of TNF- $\alpha^{+}$ $\mathrm{CD}^{+}$TILs in human CRCs. TNF- $\alpha$ was produced by 2 main cellular sources, namely TCs and macrophages. We also found weak TNF- $\alpha$ expression in some tumor cells. In contrast, fibroblasts or endothelial cells were largely TNF- $\alpha$ negative. Approximately $65 \%$ of TNF- $\alpha$-positive cells in CRC tissue were macrophages, $20 \%$ were TCs, and $9 \%$ tumor cells. High TNF- $\alpha$ expression in TCs was associated with high TNF- $\alpha$ expression in macrophages. Taken together, the 3 populations contributed $>90 \%$ of TNF- $\alpha-$ positive cells in CRC tissue.

Interestingly, the numbers of $\mathrm{TNF}-\alpha^{+} \mathrm{CD} 4^{+}$and $\mathrm{CD} 8^{+} \mathrm{TCs}$ (Supplemental Figure 5), the total amount of TNF- $\alpha^{+}$cells in CRC tissues (Figure 4, E and G), and the total TNF- $\alpha$ protein concentrations (Supplemental Figure 6) showed a strong correlation in primary tumors, while such correlation was not significant in tumor metastases (Supplemental Figure 7, A and C). In contrast, small proportions of $\mathrm{CD}^{+}$or $\mathrm{CD} 4^{+} \mathrm{TCs}$ in CRCs did not or only weakly correlated and TNF- $\alpha^{+}$cytokeratin ${ }^{+}$tumor cells even inversely correlated with TNF- $\alpha$ expression (Figure 4, F and H; Supplemental Figure 6; Supplemental Figure 7, B and D; and Supplemental Figure 8). This suggests a particularly relevant connection among TNF- $\alpha^{+}$TCs and TNF- $\alpha^{+}$stroma cells for total TNF- $\alpha$ expression in CRCs. Taken together, increased intratumoral TNF- $\alpha$ concentrations indicate accumulations of TNF- $\alpha^{+}$TA-specific TCs in CRCs.

Increased TNF- $\alpha$ concentration in CRC tissue is an independent factor of improved survival. In order to assess a potential prognostic relevance of in situ active tumor-specific TCs, we correlated TNF- $\alpha$ concentrations in a cohort of 102 patients with CRC to overall survival in comparison to other immune parameters of potential prognostic relevance, involving $\mathrm{CD}^{+}$or $\mathrm{CD} 8^{+} \mathrm{Tconv}$, Treg, and mast cell infiltrates. Besides CRCs, we analyzed cryopreserved normal colonic mucosa from all patients. 43 patients (42\%) were in International Union Against Cancer (UICC) stage II, 36 patients (35\%) were in UICC stage III, and 23 patients $(23 \%)$ were in UICC stage IV. Most of the UICC stage IV patients had liver metastases (21 patients; 91\%), followed by lung metastasis (2 patients; 9\%). Further patient characteristics and surgical procedures are shown in Table 1. Estimated median follow-up time was 135 months. Three patients were lost to follow-up after approximately 5 years. Forty-nine of ninety-nine patients (50\%) died within 10 years of follow-up, of which 38 patients died due to tumor relapse. One patient died at 11 years of follow-up. Among the 38 deceased patients, 2 were in UICC stage II ( $5 \%$ of UICC II cases), 15 were in UICC stage III (42\%) and 21 were in UICC stage IV (91\%), respectively.

The most abundant immune cell population in CRCs was CD ${ }^{+}$ TCs (79.3 cells per high-power field [HPF]), followed by mast cells (33.3 cells per HPF), CD4 ${ }^{+}$Tconv (5.2 cells per HPF), and Tregs (1.5 cells per HPF) (Table 2). CD8 ${ }^{+}$TCs (79.3 cells per HPF vs. 19.1 cells per HPF, $P=0.01)$ and Tregs $(1.5$ cells per HPF vs. 0.1 cells per 
A

TIL
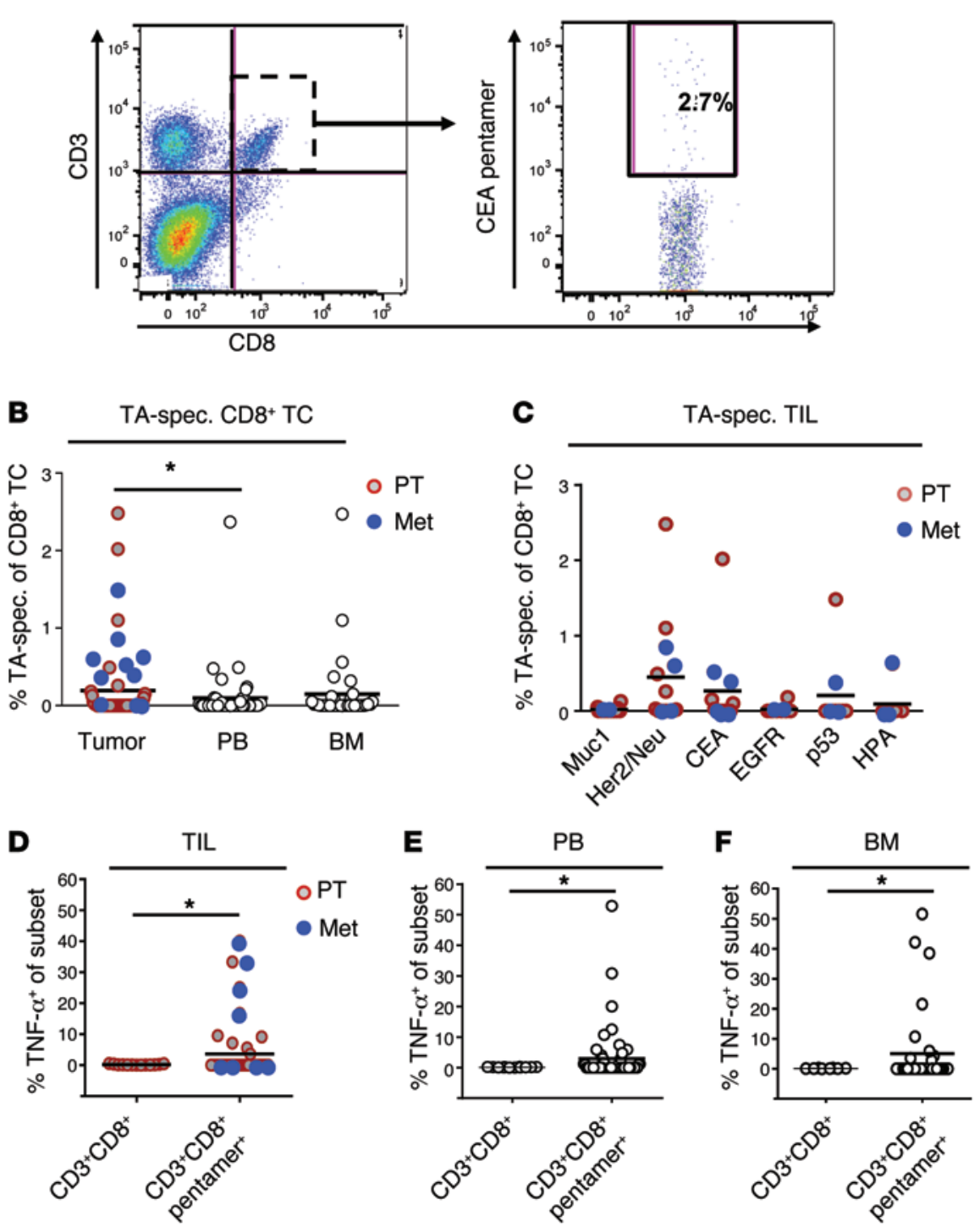

HPF, $P=0.0001)$ as well as TNF- $\alpha$ levels $(0.34 \mathrm{pg} / \mathrm{ml}$ vs. $0 \mathrm{pg} / \mathrm{ml}$; $P=0.001)$ were strongly increased in CRCs compared with normal colonic mucosa (Table 2 and data not shown), but their values showed no significant correlation among each other (Supplemental Figure 8 and data not shown).

Significantly lower $\mathrm{CD}^{+}$and Treg numbers were present at later tumor stages than at early stages $(P=0.001$, both $)$, and patients with metastatic disease had the lowest levels of $\mathrm{CD}^{+}$and Treg infiltrates ( 2.0 cells per HPF vs. 6.5 cells per $\mathrm{HPF}$ and 0.2 cells per HPF vs. 2.0 cells per HPF; $P=0.001$, both for distant metastases) (Supplemental Table 1). Accordingly, the median numbers of tumor-infiltrating $\mathrm{CD} 4^{+}$TCs and Tregs was significantly reduced in patients with cancer-related death ( 6.6 cells per HPF vs. 3.6 cells per HPF and 2.0 cells per HPF vs. 0.5 cells per HPF; $P=0.0001$, both) (Table 2). Similarly, mast cells showed a trend toward reduced numbers in advanced $\mathrm{T}$ or $\mathrm{M}$ stages (Supplemental Table 2 ), and mast cell numbers in patients who died within the observation period were significantly reduced (22.2 cells per HPF vs. 36.5 cells per HPF; $P=0.008$ ) (Table 2 ). In contrast, $\mathrm{CD}^{+} \mathrm{TC}$ infiltrates
Figure 3. TNF- $\alpha$ expression in CRC tissue is restricted to tumor-specific TCs. (A) Dot plot showing the presence of CEA-specific TCs among gated $\mathrm{CD}^{+} \mathrm{CD}^{+}{ }^{+} \mathrm{TC}$ in $\mathrm{CRC}$ tissue from one representative patient with CRC $(n=15)$. The proportion of CEA-specific CTLs among gated CD8 ${ }^{+}$TCs is indicated. (B) Proportions of TA-specific TCs among gated CD8 ${ }^{+}$TCs in primary (PT) or metastasized (Met) tumor tissue and in blood (PB; white circles) or bone marrow (white circles) of 15 patients with CRC, as determined by staining with tumor peptide-loaded HLA-A2 pentamers. Circles represent data from individual tests with all tested TAs. Mean values are indicated by black lines. ${ }^{*} P<0.05$, as determined by unpaired 2 -tailed $t$ test. (C) Proportions of tumor-infiltrating TCs from the same patients are separately shown for each tested TA. Mean values are indicated by black lines. (D-F) Proportions of TNF- $\alpha$-positive TCs among $\mathrm{CDB}^{+} \mathrm{CD} 8^{+} \mathrm{TA}$-specific (pentamer ${ }^{+}$) or unspecific (CD3+CD8 $\left.{ }^{+}\right)$TCs isolated from primary or metastasized tumor tissue (TIL) (D), blood (E), or bone marrow (F) of 14 patients with CRC. Circles represent data from individual tests with all tested TAs. Mean values are indicated by black lines. ${ }^{*} P<0.05$, as determined by unpaired 2 -tailed $t$ test.

were not significantly associated with tumor progression or metastasis (Supplemental Table 2), and intratumoral TNF- $\alpha$ levels were even increased in metastatic patients (0.5 pg/ml vs. 0.2 pg/ml; $P=0.04$; Supplemental Table 3). Still, both parameters were not altered in patients with tumor-related death (Table 2). Notably, none of the analyzed parameters (Tconv, Tregs, mast cells, TNF- $\alpha$ ) showed any considerable difference in regard to sex, age, or tumor localization (Supplemental Table 4).

We next assessed the correlation of each of these parameters with patient survival. Altogether, the disease-specific 5-year survival was $98 \%$ in UICC stage II, $67 \%$ in UICC stage III, and $13 \%$ in UICC stage IV. After 10 years, the disease-specific survival rate was $95 \%$ in UICC stage II, 58\% in UICC stage III, and 9\% in UICC stage IV, respectively. We fitted Cox regression models for the cause-specific hazards of disease-specific survival, including all tested parameters. As only 2 UICC stage II patients died from tumor progression, we did not perform a statistical analysis for patients with this stage. In UICC stage III patients, an increase in TNF- $\alpha$ concentration was statistically significantly associated with a better disease-specific survival $(P=0.05$, HR of 0.29 for $1 \mathrm{pg} / \mathrm{ml} \mathrm{TNF-} \alpha$; Table 3). In contrast, infiltrating CD4+ Tconv, $\mathrm{CD}^{+}$, or mast cells of the tumor tissue did not significantly correlate with disease-specific survival. Interestingly, in accordance with previous reports $(3,30)$, increased numbers of $\mathrm{FOXP3}{ }^{+} \mathrm{CD} 4^{+}$ Tregs were also correlated with improved survival $(P=0.02$, HR of 0.58 for 1 Treg per HPF; Table 3). For further illustration of the respective parameters, we stratified patients into groups 
Table 2. Infiltration of Tregs and CD4+ and CD8+ TCs; mast cells; and TNF- $\alpha$ and TGF- $\beta$ in CRC tissue and HT

\begin{tabular}{|c|c|c|c|c|}
\hline & $\begin{array}{c}\text { CRC } \\
\text { (median [IQR per HPF]) }\end{array}$ & $\begin{array}{c}P \text { value } \\
\text { (median [IQR per HPF]) }\end{array}$ & HT & $P$ value \\
\hline \multicolumn{5}{|l|}{$\mathrm{CD}^{+}$} \\
\hline All & $5.2(3.2-8)$ & 0.0001 & $5.3(3.7-7.1)$ & 0.056 \\
\hline Patients survived & $6.6(4.3-8.8)$ & & $5.7(4.5-8.1)$ & \\
\hline Patients with TRD & $3.6(1.9-5)$ & & $4.5(3.2-6.3$ & \\
\hline \multicolumn{5}{|l|}{$\mathrm{CD}^{+}$} \\
\hline Patients with TRD & $61.1(39.5-148)$ & & $16.6(4.8-44)$ & \\
\hline \multicolumn{5}{|l|}{ Tregs } \\
\hline All & $1.5(0.5-3)$ & 0.0001 & $0.1(0.02-0.1)$ & 0.008 \\
\hline Patients survived & $2(1.1-3.5)$ & & $0.1(0.1-0.1)$ & \\
\hline Patients with TRD & $0.5(0.2-1.4)$ & & $0.1(0-0.1)$ & \\
\hline Patients with TRD & $22.2(12.3-38.1)$ & & $58.3(47-102.9)$ & \\
\hline \multicolumn{5}{|l|}{ TNF- $\alpha$} \\
\hline All & $0.34(0.08-1.04)$ & 0.56 & $0(0-0.08)$ & 0.004 \\
\hline Patients survived & $0.34(0.07-1.02)$ & & $0(0-0.05)$ & \\
\hline Patients with TRD & $0.34(0.08-1.38)$ & & $0.08(0-0.08)$ & \\
\hline
\end{tabular}

Patient survival is based on 10 -year follow-up. Fifty patients were still alive after 10 years, and 3 patients were lost after approximately 5 years. Forty-nine patients died within 10 years, of which 38 patients died due to tumor relapse (TRD), and 11 patients died due to other causes. HT, heathy tissue; $\mathrm{IQR}$, interquartile range.

of low and high immune cell infiltration or cytokine concentration according to the respective median value. Figure 5 provides estimates of cumulative incidence curves with respect to dichotomized levels of TNF- $\alpha$, Treg, and $\mathrm{CD}_{4}^{+}$infiltration, revealing the lowest tumor-related mortality in the group of patients with elevated TNF- $\alpha$ levels. Stratification of patients into quartiles of intratumoral TNF- $\alpha$ content revealed a 10-year diseasespecific survival rate of $90 \%$ in the $>75 \%$ quartile, $77 \%$ in the $>50 \%$ quartile, and $66 \%$ in the $>25 \%$ quartile. In the small group of patients with distant metastases (UICC stage IV; $n=23$ ), none of the parameters showed a statistically significant association with disease-specific survival (data not shown).

\section{Discussion}

A protective role of endogenous tumor-specific TCs on cancer progression and patient survival has been postulated for a long time, particularly following recent reports that increased effector/memory TC infiltrates in human cancers correlate with improved patient prognosis (4). Endogenous TCs with specificity for tumor-associated antigens have been reported in the blood, bone marrow, and - with limitations - also in the tumor tissue of patients with many tumors $(10-13,40)$. Nevertheless, a direct demonstration of their activation and effector function in situ and of their impact on patient survival is still missing, which may be attributed to the lack of biomarkers of antigen-specific $\mathrm{TC}$ activity that can be assessed on stored tissue samples. In the past, the presence of type 1 and 2 TCs in tumors was determined by their expression of lineage-restricted transcription factors T-bet and GATA-3, respectively (41). However, these studies neither provided direct evidence of TC activity in situ nor did they address tumor specificity. In an alternative approach, cell surface expression of $\mathrm{CD} 107 \mathrm{a}$ has been explored as a marker for cytotoxic activity of CTLs in cryosections of CRC tumor tissue (14), but a precise distinction between intracellular and cell surface expression by immunohistochemistry is difficult, and thus CD107a staining remains a preferred marker for flow cytometric staining of freshly isolated TILs.

Although the series of individual experiments reported here are based on rather small numbers of tested samples and thus warrant further confirmation, they still provided consistent data, which overall justify our conclusion that TNF- $\alpha$ expression in CRC indicates the activity in situ of TA-specific CTLs. This can be deduced from the following observations:

Increased TNF- $\alpha$ protein expression in CRC compared with normal colonic mucosa was restricted to those patients who contained in their bone marrow or blood tumor-reactive TCs, as indicated by positive IFN- $\gamma$ ELISPOT results, while CRCs from patients who lacked such TC responses contained only minute TNF- $\alpha$ concentrations comparable to those in normal colonic mucosa. Furthermore, TNF- $\alpha$ expression in TCs ex vivo isolated from tumor tissue, blood, and bone marrow was strictly confined to the population of TA-specific TCs, as determined by HLA pentamer analysis. In addition, TNF- $\alpha$ expression reliably defined a population of activated type 1 TCs, as TNF- $\alpha$ was not expressed 
A

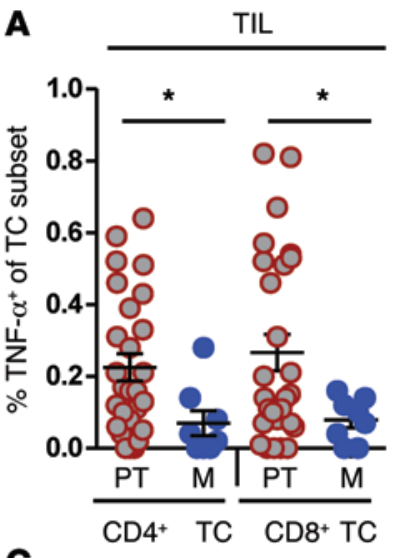

C

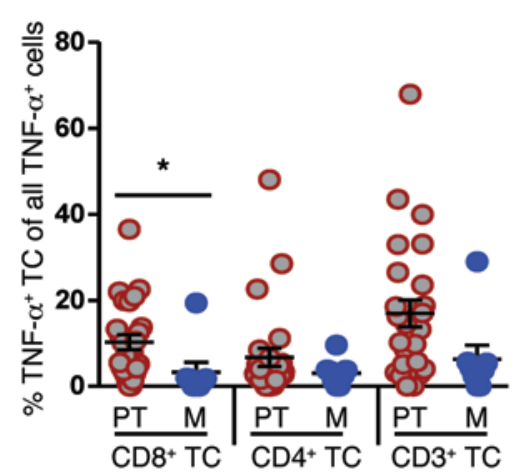

E

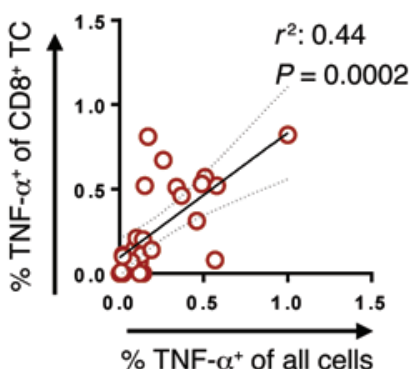

G

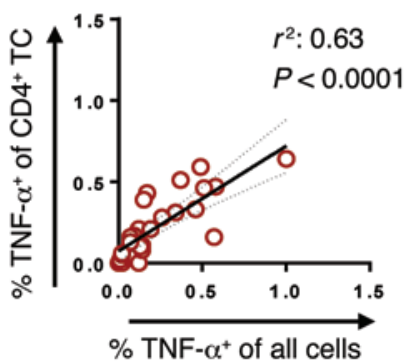

B
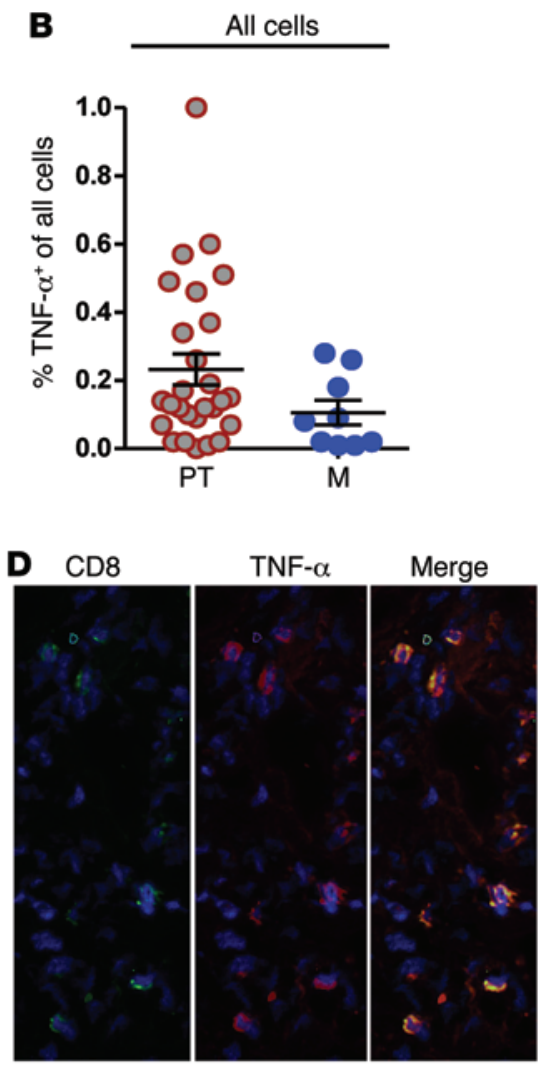

20x magnification

$\mathbf{F}$

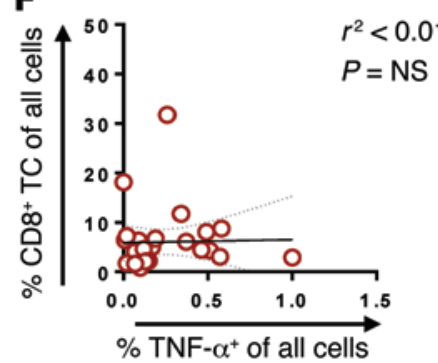

H

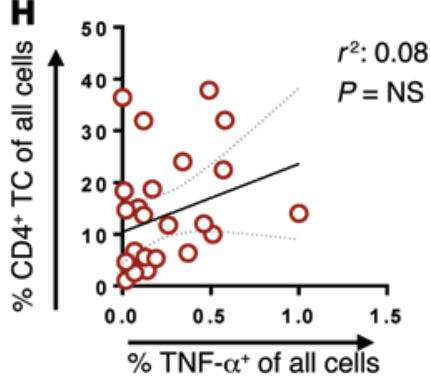

Figure 4. TNF- $\alpha$ expression in TILs correlates with total TNF- $\alpha$ expression in CRC tissue. (A-C) Proportions of TNF- $\alpha$-positive TCs among TCs (A), among total tumor-infiltrating cells (B), and among all TNF- $\alpha$-positive cells (C) in primary tumors (PT) or CRC metastases (M) of 36 patients with CRC. Each circle represents values from one patient. TNF- $\alpha$ expression in TCs is separately shown for $\mathrm{CD}^{+}, \mathrm{CD}^{+}$, and total $\mathrm{CD}^{+} \mathrm{TCs} .{ }^{*} P<0.05$, as determined by unpaired 2-tailed $t$ test. Mean \pm SEM. (D) In situ expression of TNF- $\alpha$ (red signal) by CD8 ${ }^{+}$ TILs (green signal) in a representative CRC specimen, as shown by immunofluorescence analysis of cryopreserved tissue (original magnification, $\times 20$ ). (E-H) Proportions of TNF- $\alpha$-positive TCs among $\mathrm{CD}^{+}(\mathbf{E})$ or $\mathrm{CD}^{+}(\mathbf{G})$ TILs and of total $\mathrm{CD}^{+}(\mathbf{F})$ or $\mathrm{CD}^{+}(\mathbf{H})$ among all tumor-infiltrating cells compared with the proportions of TNF- $\alpha$-positive TCs among all tumor-infiltrating cells. Circles represent individual data from 27 different primary tumors. Strength and significance of respective correlations are indicated by $r^{2}$ and $P$ values.

quently immigrate into the tumor. Indeed, since TNF- $\alpha$ was also found in pentamerbinding TCs in the blood and bone marrow, tumor-specific TC activation may also occur in lymphoid organs, such as the bone marrow, which is a well-known site for induction of tumor-specific TC responses (42).

Although we detected variable numbers of pentamer-binding CTLs in different CRCs, these were altogether enriched in tumor tissue compared with blood. This is in accordance with a previous report demonstrating that accumulation and maintenance of TCs in inflamed peripheral tissues required their interaction with cognate antigen (43). We made the observation that activated intratumoral tumor-specific CTLs were mainly found in those patients also showing activated tumor-specific CTLs in the circulation. This is in line with our observation that patients containing tumor-reactive TCs (as determined by IFN- $\gamma$ ELISPOT) showed increased levels of intratumoral TNF- $\alpha$. Still, a considerable proportion of patients with elevated frequencies of either tumor-reactive IFN- $\gamma$-secreting TCs or TNF- $\alpha$-expressing TA-specific CTLs in the blood showed no

in blood- or tumor-derived TCs after overnight resting conditions but only induced in TCs from both sources for a short period after TCR stimulation. This is in line with the reported short 2- to 4-hour duration of TNF- $\alpha$ expression after TC activation (28).

The fact that only pentamer-binding TILs expressed TNF- $\alpha$ suggests that TNF- $\alpha$ expression is triggered upon antigen recognition in situ. Nevertheless, we cannot rule out the possibility that tumor-specific TCs acquire TNF- $\alpha$ expression upon antigen-specific activation elsewhere, e.g., in lymphoid organs, and subse- increased TNF- $\alpha$ or TNF- $\alpha$-expressing TA-specific TILs in the tumors. Thus, the presence of circulating tumor-reactive TCs appears to be required but not sufficient for efficient infiltration and in situ activity of CTLs.

TNF- $\alpha$ expression was very low in the population of pentamer-negative $\mathrm{CD}^{+}$TILs, suggesting that, along with tumorspecific TCs, inactive bystander TCs also accumulate in CRCs. Bystander infiltration may be triggered by activated tumor-specific TCs, since type 1 TC-derived TNF- $\alpha$ and IFN- $\gamma$ trigger the 


\section{Table 3. Infiltration of Tregs and $C D 4^{+}$and $C D 8^{+}$TCs; mast cells; and TNF- $\alpha$ in CRC}

\begin{tabular}{lccc} 
& \multicolumn{3}{c}{ Tumor-related death (15 events) } \\
& HR $^{\mathrm{A}}$ & $\mathbf{9 5 \%} \mathrm{Cl}$ & $\boldsymbol{P}$ value \\
$\mathrm{CD}^{+}$ & 0.97 & $(0.81-1.18)$ & 0.788 \\
$\mathrm{CD}^{+}$ & 1.00 & $(0.99-1.01)$ & 0.716 \\
Tregs & 0.58 & $(0.36-0.93)$ & 0.024 \\
Mast cell & 1.00 & $(0.97-1.02)$ & 0.779 \\
TNF- $\alpha$ & 0.29 & $(0.08-0.99)$ & 0.048
\end{tabular}

ACause-specific hazard ratio. Multivariable Cox models for the cause-specific hazards for disease-specific mortality in UICC stage III patients. Hazard ratios are estimated for an increase of 1 per HPF or $1 \mathrm{pg} / \mathrm{ml}$ TNF- $\alpha$, respectively.

secretion of TH1 trophic chemokines CXCL9 and CCL10 in innate immune cells, e.g., eosinophils (44). Indeed, Pages et al. demonstrated that CXCL9 expression correlated with increased TC infiltrates and type $1 \mathrm{TC}$ signatures and with improved prognosis of patients with CRC $(4,8)$.

While all CRCs contained pentamer-binding TILs, TNF- $\alpha^{+}$ TILs were detected in only half of the tested tumors. In these, though, relatively large proportions of up to $60 \%$ expressed TNF- $\alpha$. This suggests that in some CRCs dominant regulatory mechanisms are active, either directing TC responses into other functional polarization, which renders them anergic or exhausted, or inhibiting their functional activity. We indeed found higher concentrations of TNF- $\alpha$ particularly in those tumors that contained lower levels of the TC-suppressive Treg effector cytokine, TGF- $\beta 1$ (34). Thus, increased intratumoral TNF- $\alpha$ concentrations indicate not only systemic induction of functionally potent CTL responses but also their dominance in situ over a major local immune-suppressive mechanism.

Although TNF- $\alpha$ expression in CRC tissue correlated with the presence of tumor-specific TCs, it can also be produced by other immune cells and by tumor cells. TNF- $\alpha$ expression by cells of the innate immune system, particularly macrophages, can be triggered by activated TCs in situ through CD40-CD40L interactions (45). Indeed, we detected a strong positive correlation of TNF- $\alpha$ expression by macrophages (comprising $65 \%$ of TNF- $\alpha$-secreting cells) with TNF- $\alpha$ expression in TILs, suggesting that activated TILs determine intratumoral TNF- $\alpha$ concentrations both directly through TNF- $\alpha$ secretion and indirectly through TNF- $\alpha$ induction in macrophages. Our observation that the number of TNF- $\alpha$-positive macrophages can exceed that of TNF- $\alpha$-positive TCs may be a consequence of the capacity of individual TCs to stimulate multiple macrophages and the broad abundance of tumor-associated macrophages. In contrast, TNF- $\alpha$ expression by CRC cells was rather reduced in the presence of high numbers of tumor-infiltrating, TNF- $\alpha$-expressing TCs.

Having established TNF- $\alpha$ as an indicator of in situ activity of tumor-specific TCs, we used this marker for an assessment of their impact on patient prognosis in a cohort of 102 patients with CRC who were followed for a very long observation period of more than 10 years, which considerably exceeds the follow-up periods of up to 3 years reported by previous studies. Moreover, we compared TNF- $\alpha$ with other potential prognostic factors of CRC, including infiltration with $\mathrm{CD} 4^{+}$or $\mathrm{CD} 8^{+} \mathrm{Tconv}$, Tregs, or mast cells. As the study cohort was comparably small, the most robust statements can be made on the subgroup of stage III CRC, which contributed with $38 \%$ of cases to the study cohort. In this subgroup, estimated cumulative incidence analyses suggested a reduced disease-specific mortality in patients with increased numbers of $\mathrm{CD} 4^{+}$Tconv, $\mathrm{CD} 8^{+} \mathrm{TCs}$, and Tregs, which is in accordance to published literature $(3,30)$. However, in our rather small cohort, only TNF- $\alpha$ and Tregs showed statistically marked prognostic relevance in stage III CRC in a multivariate analysis. This may be due to a different mode of TC quantification or the rather small size of our study cohort, which may have caused an underestimation of the established prognostic immune parameters $\mathrm{CD} 4^{+} \mathrm{Tconv}$ and $\mathrm{CD} 8^{+} \mathrm{TCs}$ but also underlines the potential relevance of TNF- $\alpha$ as a prognostic parameter in UICC stage III CRC, which needs to be confirmed in subsequent studies on larger patient cohorts.

The correlation of increased TNF levels with improved clinical outcome is a surprising finding, as TNF- $\alpha$ is often expressed by colorectal tumor cells and, as such, assumed to promote protumorigenic inflammation and apoptosis of TNF receptor-positive CD8 ${ }^{+}$TCs (46). A recent study that particularly focused on the impact of TNF- $\alpha$ expression in microdissected CRC cells reported that strongly increased TNF- $\alpha$ expression correlated with lymph node metastasis and reduced patient survival (47). On the other hand, TNF- $\alpha$ secreted at low levels by cells in the tumor stroma increases blood vessel permeability $(46,48)$ and, together with IFN- $\gamma$, augments TC recruitment and tumor immune control (49). A potential beneficial effect of TNF- $\alpha$ was revealed by a retrospective analysis of 40 CRC patients in who increased TNF- $\alpha$ concentrations in the sera correlated with improved survival (50). Thus, in contrast to tumor cell-derived TNF- $\alpha$, immune cell-derived TNF- $\alpha$ may exert protective effects or at least indicate protective antitumor immune responses. Accordingly, TNF- $\alpha$ concentration in total CRC tissues was determined by TNF- $\alpha$-expressing stroma cell populations but not by tumor cells, as indicated by our observation that the frequencies of TNF- $\alpha$-positive tumor cells inversely correlated with total TNF- $\alpha$ concentration.

Here, we focused on TNF- $\alpha$ as a biomarker for in situ active tumor-specific TILs, because, in our hands, TNF- $\alpha$ expression overall showed the best signal-to-noise ratio in isolated TILs, showed a favorable distinction profile between early activated and quiescent TC cultures, and was technically most suitable for combination with HLA-peptide pentamers in multicolor flow cytometry. As a consequence, our findings do not imply a superiority of TNF- $\alpha$ over other type 1 cytokines such as IFN- $\gamma$, which may as well represent a suitable biomarker for intratumoral TC activity in CRC. However, by using TNF- $\alpha$ as a functional TC marker in CRC tissue, we were able to confine the prognostic impact of TC infiltrates to the small subpopulation of TA-reactive TCs.

In conclusion, our observation that TNF- $\alpha$, as an indicator of tumor-specific CTL activity in situ, has a stronger prognostic impact than mere effector TC infiltration alone demonstrates a causal relationship among systemic TC responses, TC activity in 

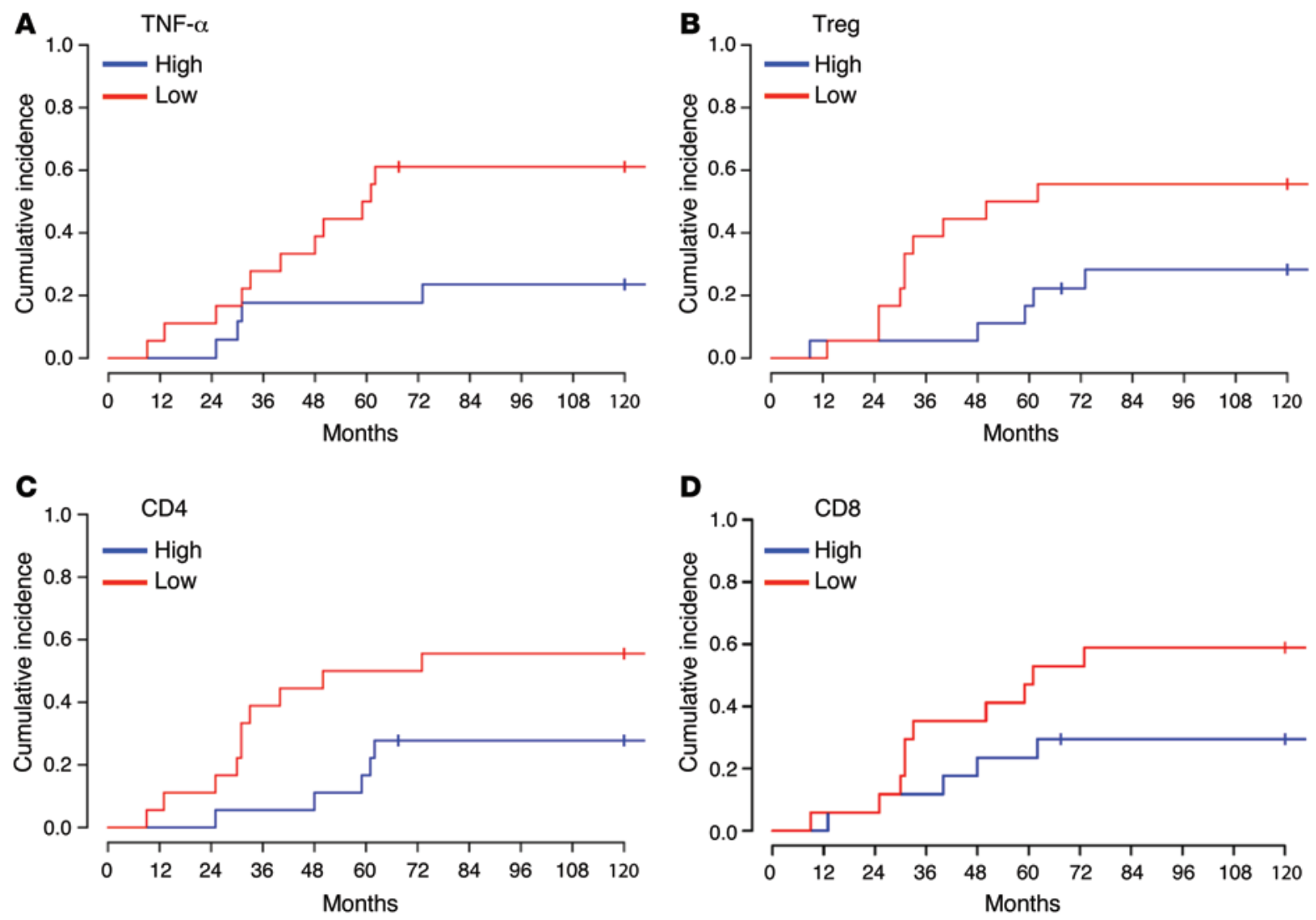

Figure 5. Cumulative incidence curves with respect to dichotomized levels of TNF- $\alpha$, Treg, and CD4+ infiltration. Cumulative incidence curves of UICC stage III patients stratified into groups with TNF- $\alpha$ concentration (median $0.51 \mathrm{pg} / \mathrm{ml}$ ) (A), tumor infiltration by Tregs (median 1.45 cells per HPF) (B), CD4 Tconv (median 5.9 cells per HPF) (C), or CD8 ${ }^{+}$TCs (median 81.7 cells per HPF) (D) above (high) or below/equal (low) median values. The respective numbers of patients at risk are given in Supplemental Table 5.

situ, and protective immune surveillance against tumor relapse in CRC. Our data thus suggest that intratumoral TNF- $\alpha$ might be a more suitable factor for prognosis prediction in patients with CRC than the quantification of effector TC infiltrates.

\section{Methods}

Patients. Blood, CRC tissue, or normal colon (distance from CRC tissue $>10 \mathrm{~cm}$ ) for experimental analysis were freshly obtained from 100 patients with primary CRC during the first tumor resection, and bone marrow samples were aspirated from the anterior iliac crest immediately after tumor resection (36). Tissue specimens were directly used for isolation of tissue-infiltrating cells and/or snap frozen in liquid nitrogen.

Assessment of prognostic factors was conducted on an additional cohort of 102 patients with histologically confirmed CRC, who underwent elective oncological resection at the Department of Surgery, University of Heidelberg, between January 1996 and January 1998. Only tissue of the primary tumor was taken. Patients with secondary malignancies in their medical history were excluded. All patients were entered in a prospectively maintained database, including data on patient baseline characteristics (age, gender, comorbidities), type of resection, and the postoperative course, with data on length of stay, in-hospital mortality, and long-term follow-up of at least 10 years. The length of follow-up was calculated from the date of operation at our institution. Occurrence of distant metastases and local recurrences, follow-up time, and rea- son for death were obtained for each patient to assess disease-specific survival. Tumor stage and grade were classified according to the seventh edition of the TNM classification of the UICC (51), and the entire population under study was stratified according to the UICC stages. Patients were staged preoperatively with colonoscopy, ultrasound of the liver, and/or CT scan and chest X-ray. Tumors located less than $16 \mathrm{~cm}$ above the anal verge were classified as rectal cancer (proved by rigid rectoscopy). Patients with UICC stage II did not receive adjuvant chemotherapy according to the recommendations that were valid at the time.

Immunofluorescence. Cryopreserved tissue was cut into 4- $\mu \mathrm{m}$ sections and fixed in $100 \%$ acetone $\left(-20^{\circ} \mathrm{C}, 5\right.$ minutes). Slides were blocked using chicken sera (5 minutes, Chicken Serum, Santa Cruz, SC-2479, 1:1,000) and incubated for 45 minutes with primary antibodies against human CD4 (polyclonal goat anti-human CD4, R\&D Systems, catalog no. AF-379-NA, 1:10) and human FOXP3 (monoclonal mouse anti-human FOXP3; AbD Serotec MCA2376, 1:100), followed by incubation with respective monoclonal secondary antibodies for 45 minutes (Alexa Fluor chicken anti-goat 488 1:200 and Alexa Fluor chicken anti-mouse 594, Life Technologies, catalog no. A11039 and A21201, respectively, 1:200). Nuclei were stained with DAPI (4 minutes, Sigma-Aldrich, 1:4,000). Finally, blocking of autofluorescence was conducted with CuSo4/NH4-Ac for 5 minutes. Analysis was performed with a fluorescence microscope (Axioplan 2, Zeiss).

Immunohistochemistry. 4- $\mu \mathrm{m}$ cryosections were fixed with $2 \%$ paraformaldehyde (Roth) for 10 minutes. Slides were blocked for 15 
minutes using a solution of $1 \mathrm{ml}$ albumin, $1 \mathrm{ml}$ huIgG (Baxter), $3 \mathrm{ml}$ PBS (PAA Laboratories), and $50 \mu \mathrm{g}$ Triton 10\% (Sigma-Aldrich). For CD8 and mast cell staining, the slides were incubated with mouse anti-human (clone 17147, Abcam) and mouse anti-human tryptase antibody (MS-1216 Lab Vision) (both 1:5,000) for 60 minutes, and polyclonal biotin-conjugated goat anti-mouse antibody (Dianova, catalog no. 115-065-044, Jackson ImmunoResearch) (1:200) was used as a secondary antibody (60 minutes). Finally, the EnVision peroxidase Kit (Dako) was used according to the manufacturer's protocol to visualize stained cells. Negative control studies were performed by omitting primary antibodies. Ten randomly selected HPFs $\left(1 \mathrm{HPF}=0.138 \mathrm{~mm}^{2}\right)$ per slide and 2 slides per patient were analyzed independently by 2 investigators, and respective countings were averaged in each case.

PBMC and TIL isolation. All blood samples were subjected to Ficoll gradient centrifugation (Biochrom) (36) to collect mononuclear cells. Tumor-infiltrating cells were obtained from freshly resected tumor samples, which were immediately placed in ice-cold PBS. The tumors were cut into small pieces and triturated in ice-cold PBS and then filtered through 100- $\mu \mathrm{m}$ cell strainers and subsequently $40-\mu \mathrm{m}$ cell strainers (Becton Dickinson). The filtrates were collected and centrifuged at $35 \mathrm{~g}$ for 3 minutes.

Cell culture. For use in IFN- $\gamma$ ELISPOT and cytokine capture assays, TCs were cultured for 7 days in RPMI medium (Gibco) containing $10 \%$ AB sera (PromoCell), $100 \mathrm{U} / \mathrm{ml} \mathrm{IL-2} \mathrm{(PromoCell),} \mathrm{and} 60 \mathrm{U} / \mathrm{ml}$ IL-4 (PromoCell). Afterward, TCs were transferred into cytokinefree media for 12 hours and subsequently purified by the T Cell Negative Isolation Kit (Invitrogen). DCs were generated as described previously (36). In brief, plastic-adherent mononuclear cells were cultured for 7 days in serum-free X-VIVO 20 medium (BioWhittaker) supplemented with $50 \mathrm{ng} / \mathrm{ml}$ rhuGM-CSF (Essex Pharma) and 1,000 U/ml IL-4 (PromoCell). DCs were enriched using anti-CD19, antiCD3, and anti-CD56-coupled magnetic beads (Dynal). For antigen presentation, DCs were pulsed overnight with test antigens $(200 \mu \mathrm{g}$ antigen per $10^{6}$ cells per $\mathrm{ml}$ ) in cytokine-free X-VIVO 20.

Antigens. As test TAs, we used synthetic long peptides derived from 9 different TAs that we have tested previously in patients with CRC. Human IgG was used as negative control antigen. In some cases, DCs were pulsed with lysates of autologous colorectal tumor cells as a source of TAAs or with lysates of autologous PBMCs or normal colonic mucosa as a source of control antigen, as described previously (36).

IFN- $\gamma$ ELISPOT assay. ELISPOT assays were done as described previously (36) with few modifications. In short, antigen-pulsed DCs were incubated with autologous TCs (1:5 ratio) for 40 hours in ELISPOT plates. IFN- $\gamma$ spots were measured using KS ELISPOT software (Zeiss). Individuals were considered as responders if spot numbers in triplicate test wells were at least 1.4-fold and also significantly (2-sided Student's $t$ test, $P<0.05$ ) higher than in negative control wells. The frequencies of tumor-reactive TCs in responders were calculated as follows: (mean spots in test wells - mean spots in control wells)/TC numbers per well.

Cytokine capture assay. The cytokine capture assay was performed using TNF- $\alpha$ and IFN- $\gamma$ Secretion Assay Detection Kits (Miltenyi Biotec $\mathrm{GmbH}$ ) according to the manufacturer's instructions, with some modifications. In short, antigen-pulsed DCs were incubated with autologous TCs (1:5 ratio) for 12 hours. Unspecific binding was blocked by preincubation of the cells for 10 minutes on ice with huIgG (Kiovig,
$1.7 \mathrm{mg} / \mathrm{ml}$ ). Then, the cells were labeled with the respective TNF- $\alpha$ and IFN- $\gamma$ catch reagents provided in the kits and were further incubated for 45 minutes at $37^{\circ} \mathrm{C}$, with tilting and rotation to allow secretion of the cytokines. The captured cytokines were detected by staining with PE-conjugated IFN- $\gamma$ - and APC-conjugated TNF- $\alpha$-specific antibodies (Detection Reagents) included in the kits. Dead cells were excluded by staining with LIVE/DEAD Fixable Yellow Dead Cell Stain (Life Technologies $\mathrm{GmbH}$; 1:1,000), followed by TC subset staining with anti-human CD4-PerCp-Cy5.5 and CD8-V450 mAbs (clones RPA-T4 and RTP-T8, respectively, BD Pharmingen).

Flow cytometry and pentamer staining. Stainings of PBMCs and TILs were conducted with $5 \times 10^{5}$ to $10 \times 10^{5}$ vital cells each. In most cases, isolated TCs were immediately processed for assessment of phenotype and cytokine expression. In some cases, TILs were rested overnight in RPMI medium containing $10 \% \mathrm{AB}$ sera but no cytokines before analysis of baseline TNF- $\alpha$ expression. Unspecific binding was blocked by preincubation of cells for 30 minutes with huIgG (Endobulin, $2.5 \mathrm{mg} / \mathrm{ml}$, Baxter Oncology), and dead cells were excluded by staining with EMA according to the manufacturer's protocol (Invitrogen). TCs in PBMC or TIL cultures were quantified by staining with respective fluorochrome-conjugated mouse anti-human CD3, CD4, and CD8 mAbs (clones UCHT1, RPA-T4, and RPA-T8, respectively; all from BD Pharmingen; 1:10). For epithelial and tumor cell staining, a directly conjugated pan-cytokeratin antibody (clone C11, Santa Cruz) was used. For intracellular TNF- $\alpha$ staining, cells were fixed in $100 \mu \mathrm{l}$ Fixation/Permeabilization Buffer (eBioscience), washed, and stained with APC-conjugated anti-TNF- $\alpha$ mAb (clone mAB11, BD Pharmingen, 1:10). Isotype control stainings were performed using respectively conjugated $\operatorname{IgG}_{1}$ $\kappa$-isotype antibodies (clone MOPC21, BD Pharmingen).

HLA typing was done using mouse anti-human monoclonal HLA-A0201-specific antibody (clone BB7.2; produced in-house) and FITC-labeled goat anti-mouse IgG secondary antibody (BD Pharmingen, catalog no. 555988) (15). For HLA pentamer stainings, HLA-A2+ PBMCs were blocked with polyclonal human immunoglobulin (Endobulin, $2.5 \mathrm{mg} / \mathrm{ml}$, Baxter Oncology) and incubated with the following mouse anti-human monoclonal antibodies: anti-CD3-FITC and antiCD8-Pacific Blue (clone HIT3a and RPA-T8, respectively, both 1:10, BD Pharmingen), supplemented with $2.5 \mu \mathrm{g} / \mu \mathrm{l}$ ethidium monoazide bromide for live cell gating for 30 minutes on ice. In addition, HLA-A2 TAA-specific Pro5 pentamers (Proimmune) coupled to PE were added to each sample, respectively. The following epitopes were tested: Muc1 LLLLTVLTV, Her2/neu KIFGSLAFL, CEA YLSGANLNL, EGFR KLFGTSGQKT, p53 STPPPGTRV, Hepa1 LLLGPLGPL, HIV-gag SLYNTVATL (ProImmune). Acquisition was performed with BD FACSCanto (BD). Data analyses were performed with FlowJo software (Tree Star). In some cases, PBMCs or TILs were polyclonally activated before flow cytometric analysis, as described previously (52), by overnight incubation with anti-human CD3/CD28 Dynabeads (TC expander, Dynal) at a bead/cell ratio of 3:1. Dynabeads were retracted from the cells afterward using a magnetic particle concentrator (Invitrogen).

ELISA. TNF- $\alpha$ protein concentration in tumor or normal tissue lysates was quantified with the Quantakine HS ELISA Kit (with sensitivity of $0.191 \mathrm{pg} / \mathrm{ml}$ ) (R\&D Systems) according to the manufacturer's protocol. The absorbance was detected using a microplate reader at $490 \mathrm{~nm}$.

Statistics. Distributions of continuous variables were described by their medians and ranges. Two group comparisons were done using 
Wilcoxon's rank-sum test for unpaired data and Wilcoxon's signedrank test for paired data. Categorical variables were characterized by counts and proportions and compared using Fisher's exact test. Results of experimental analyses were compared using paired and unpaired 2-tailed $t$ tests. Correlation analyses were performed based on Pearson's correlation coefficient. Median follow-up time was estimated with the reverse Kaplan-Meier method (53). The proportional hazards regression model of Cox was used to investigate the influence of the 5 biomarkers on overall survival. To assess disease-specific survival, Cox models for cause-specific hazards were applied. Multivariable regression models were always used. A result was considered as statistically significant if the corresponding $P$ value was less than 0.05. For exploratory univariable survival analysis, the 5 biomarkers were dichotomized at median level. Cumulative incidence curves were calculated by the Aalen-Johansen estimate. The statistical analyses were carried out using software packages $\mathrm{R}$ (version 3.0.2, R Core Team 2013), JMP (SAS Institute), and SPSS (SPSS Inc.).
Study approval. All patient samples were obtained after each patient provided informed consent and upon approval of the study protocol by the ethics committee of the University Medical Center, Heidelberg.

\section{Acknowledgments}

This work was supported by Deutsche Forschungsgemeinschaft (SFB938 and KFO227).

Address correspondence to: Philipp Beckhove, Translational Immunology Unit, German Cancer Research Center, Im Neuenheimer Feld 460, 69120 Heidelberg, Germany. Phone: 49.6221.56.5466; E-mail: p.beckhove@dkfz.de.

Khashayarsha Khazaie's present address is: Departments of Immunology and Surgery, Mayo Clinic College of Medicine and Graduate School, Rochester, Minnesota, USA.
1. Mensink PB, Kolkman JJ, Van Baarlen J, Kleibeuker JH. Change in anatomic distribution and incidence of colorectal carcinoma over a period of 15 years: clinical considerations. Dis Colon Rectum. 2002;45(10):1393-1396.

2. Weitz J, Koch M, Debus J, Hohler T, Galle PR, Buchler MW. Colorectal cancer. Lancet. 2005;365(9454):153-165.

3. Galon J, et al. Type, density, and location of immune cells within human colorectal tumors predict clinical outcome. Science. 2006;313(5795):1960-1964.

4. Pages F, et al. Effector memory T cells, early metastasis, and survival in colorectal cancer. N Engl JMed. 2005;353(25):2654-2666.

5. Funada Y, Noguchi T, Kikuchi R, Takeno S, Uchida Y, Gabbert HE. Prognostic significance of $\mathrm{CD}^{+} \mathrm{T}$ cell and macrophage peritumoral infiltration in colorectal cancer. Oncol Rep. 2003;10(2):309-313.

6. Naito Y, et al. $\mathrm{CD}^{+} \mathrm{T}$ cells infiltrated within cancer cell nests as a prognostic factor in human colorectal cancer. Cancer Res. 1998;58(16):3491-3494.

7. Ropponen KM, Eskelinen MJ, Lipponen PK, Alhava E, Kosma VM. Prognostic value of tumour-infiltrating lymphocytes (TILs) in colorectal cancer. J Pathol. 1997;182(3):318-324.

8. Pages F, et al. In situ cytotoxic and memory $\mathrm{T}$ cells predict outcome in patients with early-stage colorectal cancer. J Clin Oncol. 2009;27(35):5944-5951.

9. Halama N, et al. Localization and density of immune cells in the invasive margin of human colorectal cancer liver metastases are prognostic for response to chemotherapy. Cancer Res. 2011;71(17):5670-5677.

10. Sommerfeldt $\mathrm{N}$, et al. Heparanase: a new metastasis-associated antigen recognized in breast cancer patients by spontaneously induced memory T lymphocytes. Cancer Res. 2006;66(15):7716-7723.

11. Choi C, et al. Enrichment of functional CD8 memory T cells specific for MUC1 in bone marrow of patients with multiple myeloma. Blood. 2005;105(5):2132-2134.

12. Schmitz-Winnenthal FH, et al. High frequencies of functional tumor-reactive $\mathrm{T}$ cells in bone marrow and blood of pancreatic cancer patients. Cancer Res. 2005;65(21):10079-10087.

13. Sommerfeldt N, Schutz F, Sohn C, Forster J, Schirrmacher V, Beckhove P. The shaping of a polyvalent and highly individual T-cell repertoire in the bone marrow of breast cancer patients. Cancer Res. 2006;66(16):8258-8265.

14. Koch M, et al. Tumor infiltrating T lymphocytes in colorectal cancer: Tumor-selective activation and cytotoxic activity in situ. Ann Surg. 2006;244(6):986-992.

15. Bonertz A, et al. Antigen-specific Tregs control $\mathrm{T}$ cell responses against a limited repertoire of tumor antigens in patients with colorectal carcinoma. J Clin Invest. 2009;119(11):3311-3321.

16. Mlecnik B, et al. Histopathologic-based prognostic factors of colorectal cancers are associated with the state of the local immune reaction. JClin Oncol.2011;29(6):610-618.

17. Nagorsen D, et al. Differences in T-cell immunity toward tumor-associated antigens in colorectal cancer and breast cancer patients. Int J Cancer. 2003;105(2):221-225.

18. Nummer $\mathrm{D}$, et al. Role of tumor endothelium in $\mathrm{CD}^{+} \mathrm{CD}^{+} 5^{+}$regulatory $\mathrm{T}$ cell infiltration of human pancreatic carcinoma. J Natl Cancer Inst. 2007;99(15):1188-1199.

19. Homey B, Muller A, Zlotnik A. Chemokines: agents for the immunotherapy of cancer? Nat Rev Immunol. 2002;2(3):175-184.

20. Gershon RK, Kondo K. Infectious immunological tolerance. Immunology. 1971;21(6):903-914.

21. Zou W. Regulatory T cells, tumour immunity and immunotherapy. Nat Rev Immunol. 2006;6(4):295-307.

22. Khazaie $\mathrm{K}$, et al. The significant role of mast cells in cancer. Cancer Metastasis Rev. 2011;30(1):45-60.

23. Robson H, Anderson E, James RD, Schofield PF. Transforming growth factor $\beta 1$ expression in human colorectal tumours: an independent prognostic marker in a subgroup of poor prognosis patients. Br J Cancer. 1996;74(5):753-758.

24. Umansky V, Sevko A. Tumor microenvironment and myeloid-derived suppressor cells. Cancer Microenviron. 2013;6(2):169-177.
25. Zou W, Chen L. Inhibitory B7-family molecules in the tumour microenvironment. Nat Rev Immunol. 2008;8(6):467-477.

26. Berinstein NL, et al. First-in-man application of a novel therapeutic cancer vaccine formulation with the capacity to induce multi-functional T cell responses in ovarian, breast and prostate cancer patients. J Transl Med. 2012;10:156.

27. Seder RA, Darrah PA, Roederer M. T-cell quality in memory and protection: implications for vaccine design. Nat Rev Immunol. 2008;8(4):247-258.

28. Han Q, Bagheri N, Bradshaw EM, Hafler DA, Lauffenburger DA, Love JC. Polyfunctional responses by human $\mathrm{T}$ cells result from sequential release of cytokines. Proc Natl Acad Sci U S A 2012;109(5):1607-1612.

29. Betts G, et al. Suppression of tumour-specific CD4(+) T cells by regulatory T cells is associated with progression of human colorectal cancer. Gut. 2012;61(8):1163-1171.

30. Salama $\mathrm{P}$, et al. Tumor-infiltrating $\mathrm{FOXP3}^{+} \mathrm{T}$ regulatory cells show strong prognostic significance in colorectal cancer. JClin Oncol. 2009;27(2):186-192.

31. Valencia X, Stephens G, Goldbach-Mansky R, Wilson M, Shevach EM, Lipsky PE. TNF downmodulates the function of human $\mathrm{CD} 44^{+} \mathrm{CD} 25^{\mathrm{hi}}$ T-regulatory cells. Blood. 2006;108(1):253-261.

32. Ling KL, et al. Increased frequency of regulatory $\mathrm{T}$ cells in peripheral blood and tumour infiltrating lymphocytes in colorectal cancer patients. Cancer Immun. 2007;7:7.

33. Blatner NR, et al. Expression of ROR $\gamma \mathrm{t}$ marks a pathogenic regulatory $\mathrm{T}$ cell subset in human colon cancer. Sci Transl Med. 2012;4(164):164ra159.

34. Domschke C, et al. Intratumoral cytokines and tumor cell biology determine spontaneous breast cancer-specific immune responses and their correlation to prognosis. Cancer Res. 2009;69(21):8420-8428.

35. Feuerer M, et al. Bone marrow as a priming site for T-cell responses to blood-borne antigen. Nat Med.2003;9(9):1151-1157.

36. Feuerer M, et al. Therapy of human tumors in NOD/SCID mice with patient-derived reacti- 
vated memory $\mathrm{T}$ cells from bone marrow. Nat Med. 2001;7(4):452-458.

37. Schmitz-Winnenthal $\mathrm{H}$, et al. Chronic pancreatitis is associated with disease-specific regulatory T-cell responses. Gastroenterology. 2010;138(3):1178-1188.

38. Muller-Berghaus J, et al. Melanoma-reactive T cells in the bone marrow of melanoma patients: association with disease stage and disease duration. Cancer Res. 2006;66(12):5997-6001.

39. Aggarwal BB. Signalling pathways of the TNF superfamily: a double-edged sword. Nat Rev Immunol. 2003;3(9):745-756.

40. Beckhove $P$, et al. Specifically activated memory $T$ cell subsets from cancer patients recognize and reject xenotransplanted autologous tumors. JClin Invest. 2004;114(1):67-76.

41. De Monte L, et al. Intratumor T helper type 2 cell infiltrate correlates with cancer-associated fibroblast thymic stromal lymphopoietin production and reduced survival in pancreatic cancer. J Exp Med. 2011;208(3):469-478.

42. Schirrmacher V, Feuerer M, Fournier P, Ahlert
T, Umansky V, Beckhove P. T-cell priming in bone marrow: the potential for long-lasting protective anti-tumor immunity. Trends Mol Med. 2003;9(12):526-534.

43. Marelli-Berg FM, Cannella L, Dazzi F, Mirenda V. The highway code of $\mathrm{T}$ cell trafficking. J Pathol. 2008;214(2):179-189.

44. Liu LY, Bates ME, Jarjour NN, Busse WW, Bertics PJ, Kelly EA. Generation of Th1 and Th2 chemokines by human eosinophils: evidence for a critical role of TNF- $\alpha$. JImmunol. 2007;179(7):4840-4848.

45. Kiener PA, Moran-Davis P, Rankin BM, Wahl AF, Aruffo A, Hollenbaugh D. Stimulation of CD40 with purified soluble gp39 induces proinflammatory responses in human monocytes. Jimmunol. 1995;155(10):4917-4925.

46. Balkwill F. Tumour necrosis factor and cancer. Nat Rev Cancer. 2009;9(5):361-371.

47. Grimm M, et al. Tumor necrosis factor-alpha is associated with positive lymph node status in patients with recurrence of colorectal cancer-indications for anti-TNF- $\alpha$ agents in cancer treatment. Cell Oncol (Dordr). 2011;34(4):315-326.
48. Johansson A, Hamzah J, Payne CJ, Ganss R. Tumor-targeted TNF $\alpha$ stabilizes tumor vessels and enhances active immunotherapy. Proc Natl Acad Sci U S A. 2012;109(20):7841-7846.

49. Muller-Hermelink N, et al. TNFR1 signaling and IFN- $\gamma$ signaling determine whether T cells induce tumor dormancy or promote multistage carcinogenesis. Cancer Cell. 2008;13(6):507-518.

50. Evans C, et al. The correlation between colorectal cancer rates of proliferation and apoptosis and systemic cytokine levels; plus their influence upon survival. Br JCancer. 2006;94(10):1412-1419.

51. Edge SB, Byrd DR, Compton CC, Fritz AG, Greene FL, Trotti AE, eds. AJCC Cancer Staging Handbook. 7th ed. New York, New York, USA: Springer; 2010.

52. Xydia M, Ge Y, Quitsch U, Beckhove P. CD4OL co-stimulation from $\mathrm{CD} 8^{+}$to $\mathrm{CD} 4^{+}$effector memory T cells supports $\mathrm{CD} 4^{+}$expansion. Immunol Cell Biol. 2011;89(6):670-680.

53. Schemper M, Smith TL. A note on quantifying follow-up in studies of failure time. Control Clin Trials. 1996;17(4):343-346. 